\title{
Targeting the energy-coupling factor (ECF) transporters: identification of new tool compounds
}

Eleonora Diamanti, ${ }^{a \mathrm{a}, \mathrm{b}}$ Inda Setyawati, ${ }^{\mathrm{cc}, \mathrm{d}}$ Spyridon Bousis, ${ }^{\mathrm{a}, \mathrm{b}}$ Paulo C. T. Souza, ${ }^{\mathrm{e}, \mathrm{f}}$ Leticia M. Gómez, ${ }^{\mathrm{b}}$ Lotteke J.Y.M. Swier, ${ }^{c}$ Jörg Haupenthal, ${ }^{a}$ Paddy S. Gibson, ${ }^{\mathrm{g}}$ Carsten Volz, ${ }^{\mathrm{h}}$ Weronika K. Stanek, ${ }^{\mathrm{c}}$ Manuel Jäger, ${ }^{\mathrm{b}}$ Jennifer

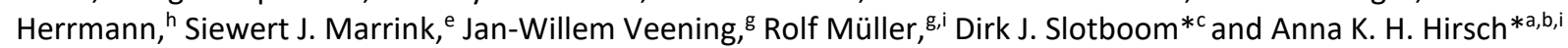

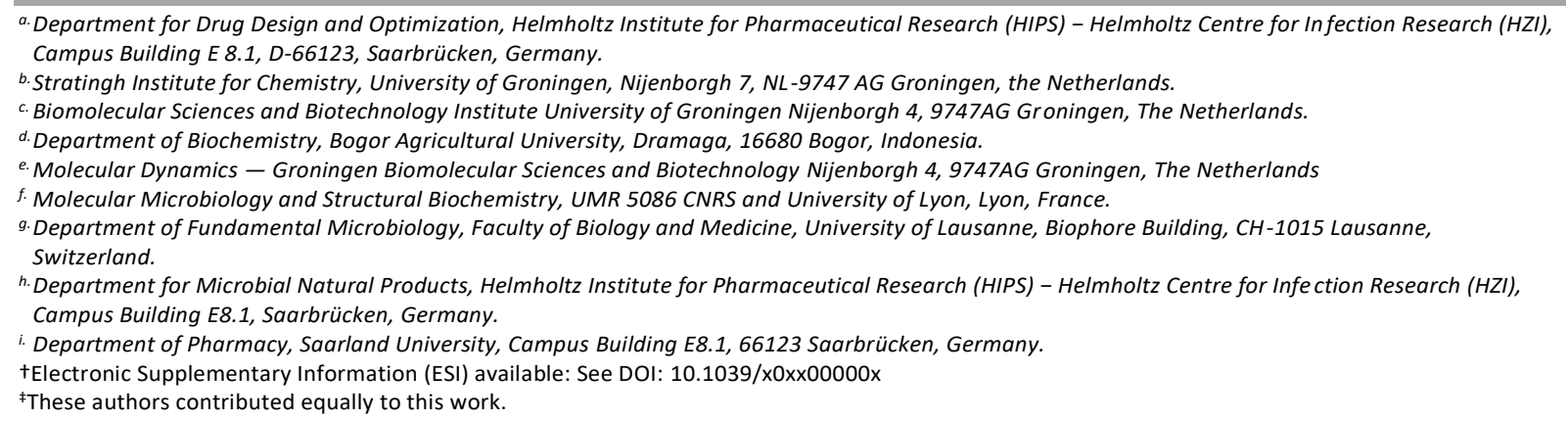

\begin{abstract}
The energy-coupling factor (ECF) transporters are a family of transmembrane proteins involved in the uptake of vitamins in a wide range of bacteria. Inhibition of the activity of these proteins could reduce the viability of pathogens that depend on vitamin uptake. Their central role in the metabolism of bacteria and absence in humans make the ECF transporters a potential antibacterial target, which can be further investigated making use of a selective chemical probe. Here, we report on the virtual screening, design, synthesis, structure-activity relationships (SARs) and coarse-grained molecular dynamics simulations of the first class of inhibitors of the ECF transporters. We investigated the mechanism of action of this chemical class and profiled the best hit compounds regarding their pharmaceutical properties. The optimized hit has a minimum inhibitory concentration (MIC) value of $2 \mu \mathrm{g} / \mathrm{mL}$ against Streptococcus pneumoniae, which opens up the possibility to use this chemical class to investigate the role of the ECF transporters in health and disease.
\end{abstract}

\section{Introduction}

Energy-coupling factor (ECF) transporters are a recently discovered subclass of the superfamily of adenosine 5'triphosphate (ATP)-binding cassette $(A B C)$ transporters. While $A B C$ transporters are present in all prokaryotic and eukaryotic species, ${ }^{1-4}$ mediating uptake or extrusion of compounds into and from cells, the ECF transporters are absent in humans but present in approximately $50 \%$ of prokaryotic species. This class of proteins mediates the uptake of essential micronutrients such as water-soluble vitamins (e.g., folate, ${ }^{5}$ riboflavin, ${ }^{6}$ cobalamin, ${ }^{7}$ biotin, ${ }^{8}$ niacin, ${ }^{9}$ thiamine, ${ }^{10}$ pantothenate $\left.{ }^{11}\right)$ and metal cations $\left(\mathrm{Ni}^{2+} \text { and } \mathrm{Co}^{2+}\right)^{12,13}$ into bacteria and archaea. They are widely distributed in the Firmicutes phylum of Gram-positive species ${ }^{14}$ and contribute to the survival and growth of the bacteria. ${ }^{15}$

The ECF transporters are transmembrane proteins consisting of two modules: an integral membrane domain dedicated to the binding of a single substrate (the S-component), and an ECF module formed by an integral membrane protein, the T-component (EcfT) and two intracellular ATPases (EcfA and EcfA', Fig. 1). ECF transporters are classified into two groups, group-I and $-1 \mathrm{I} .{ }^{16}$ In group I, the ECF module interacts exclusively with a single "dedicated" S-component, whereas the module in group-II interacts with different ones. In group-II, the same ECF module can associate with distinct S-components, opening up the possibility to block the uptake of several vitamins with a single inhibitor.

A recently proposed mechanism of transport postulates that the S-component can dissociate from the ECF module, which allows for exposure of the substrate binding pocket to the extracellular environment. ${ }^{17,18}$ After binding, the substrate is buried inside a cavity, and the S-component can rotate (topple over) by $\sim 90$ degrees in the plane of the membrane. In the toppled state, the S-component binds to the ECF module, which leads to the release of the substrate inside the cytosol. ${ }^{19}$ Subsequent pinching of two long alpha helical elements, the coupling helices, of the EcfT subunit is predicted to occur by hydrolysis of ATP, and causes re-orientation and dissociation of the substrate-free S-component, marking the end of a transport cycle. Hence, inhibitors that specifically block the movements taking place 
in the ECF module, in particular the coupling helices, could impact the uptake of various vitamins (mediated by different S-components) by the bacterium.

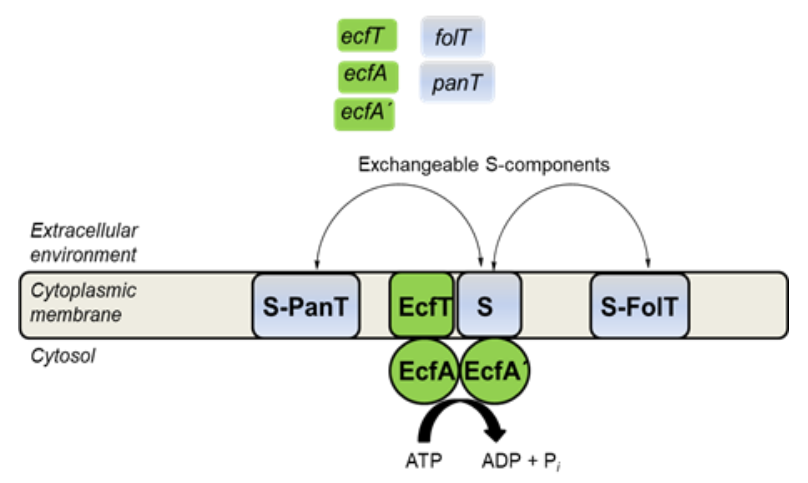

Fig. 1 Architecture of ECF group-II transporters. In green ECF module (EcfT, EcfA and EcfA') and in blue the S-components (e.g., the ones specific for folic acid (FoIT), panthotenate (PanT)). The S-components interact with a shared ECF module. Genes coding for Scomponents are scattered around the chromosome, while the ECF-module genes are usually clustered.

Given that this inhibition likely affects growth and survival, it constitutes a new avenue for intervention in bacterial infections. Despite the important functions of ECF transporters, no inhibitor targeting the unique transport mechanism has been reported to date, only substrate-mimicking competitive inhibitors for the S-component ThiT from Lactococcus lactis have been reported. ${ }^{20-22}$

Here, we describe the discovery of unexplored chemical structures, with compound $\mathbf{2 0}$ (Table 1) as the best in the series, as the first inhibitors of ECF-transporter activity. We further show that this compound is able to inhibit the uptake of more than one vitamin and may be used in vitro to explore the function of ECF transporters.

\section{Results and discussion}

The absence of known inhibitors, as well as the lack of a robust high-throughput activity assay hampered a medicinal-chemistry exploration of ECF transporters. Despite the relatively low resolution (3.0 A) of the available crystal structure of a folate-specific transporter ECF-FolT from Lactobacillus delbrueckii (PDB ID 5JSZ), ${ }^{23}$ and the fact that we are dealing with transmembrane protein with a highly dynamic mechanism of action, we decided to perform a structure-based virtual screening (SBVS) as the only viable hit-identification approach.

Here, we report on the SBVS campaign using the X-ray crystal structure of ECF-FolT (PDB ID 5JSZ), ${ }^{23}$ which yielded compounds $\mathbf{1}$ and $\mathbf{2}$ as inhibitors of ECF-mediated vitamin uptake (Fig. 2). We selected the structure of the transporter from $L$. delbrueckii, because for this protein a robust, albeit low throughput activity assay is available.

The first step in the identification of new hits for the ECF transporters was the selection of druggable pockets, for which we used a fully automated algorithm, DoGSiteScorer, (Fig. S1). ${ }^{24}$ A strategic localization near the predicted surface of the membrane, where the coupling helices of the EcfT component are expected to change conformation during transport, and a score of 0.81 , guided us to select the so-called P2 pocket as the most promising allosteric binding site (Fig. S2). Compounds binding to this pocket were expected to interfere with the transport cycle and inhibit uptake of all substrates of the ECF transporter in question. We performed the virtual screening on the Express Collection of Princeton BioMolecular Research (1.3 million compounds) using the KNIME Analytics Platform ${ }^{25}$ and $^{2}$ LeadIT ${ }^{26}$ with the scoring function HYDE implemented in SeeSAR ${ }^{27}$ and followed a typical workflow (details are provided in the Supplementary Information), applying a range of filters to focus on drug-like compounds and exclude frequent hitters. ${ }^{28-30}$ The final selection of the 100 top-ranked compounds included visual inspection, assessment of the docked pose and estimation of the drug-like properties. As a result, we selected twelve distinct molecular scaffolds (1-12, Fig. 2 and S3) for biochemical screening. A transport-activity assay on purified ECF-FolT2 reconstituted in proteoliposomes using radiolabeled folate showed that 1 and $\mathbf{2}$ are able to reduce translocation of the vitamin across the membrane with $I_{50}$ values of $282 \mu \mathrm{M}$ and $1.2 \mathrm{mM}$, respectively. Hit 2, despite its weak inhibitory activity, has a comparable ligand efficiency to hit 1 and it may be bound in the same region, but it suffers from solubility issues given its zwitterionic nature. 


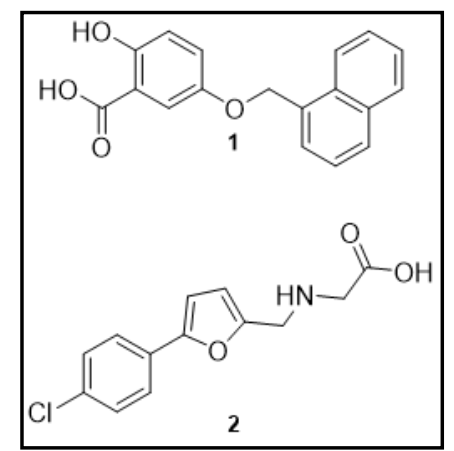

Fig. 2 Chemical structures of hit compounds (1 and $\mathbf{2}$ ) identified through structure-based virtual screening using the crystal structure of Lactobacillus delbrueckii ECF-FolT2 (PDB ID 5JSZ). ${ }^{23}$

As a result, we selected hit $\mathbf{1}$ for our study because of its chemical tractability and more favorable physicochemical properties.

The fragment-sized hit compound 1 with a MW 294.3, cLogP 3.5 and ligand efficacy (LE) of 0.22 was selected as starting point for subsequent hit validation through resynthesis, retesting and evaluation of its in vitro pharmacokinetic properties. Assessment of the inhibition of the ECF transporters, along with in vitro metabolism and cytotoxicity studies supported the potential of this chemotype initially as chemical probe and ultimately for the development of agents that target the ECF transporters.

\section{Biochemical assay to validate HIT 1}

This assay was performed by the purification and reconstitution into proteoliposomes of ECF-FolT2 and ECF-PanT, which share the same ECF module, but use different S-components, for folic acid and pantothenate, respectively. At an inhibitor concentration of $5 \mathrm{mM}$, compound 1 was able to completely inhibit the transport of both folate and pantothenate, indicating that it binds to the shared ECF module. To further validate that the inhibition values of $\mathbf{1}$ are not due to an artifact like disruption of the lipid bilayer or chelation of $\mathrm{Mg}^{2+}$, which is crucial for the hydrolysis of ATP, or direct interference with the hydrolysis of ATP, we used the ABC transporter OpuA from L. lactis, a classical ABC transporter that mediates the uptake of glycine betaine, with membrane domains unrelated to ECF transporters, as negative control. Since the ATP hydrolysis sites are well-conserved among all ABC transporters, OpuA can be used to evaluate the selectivity of the compounds. OpuA was purified and reconstituted in liposomes. The uptake of glycine-betaine into the proteoliposomes was not affected by the presence of 1, providing the first indication that our compound neither inhibits the hydrolysis of ATP by ABC transporters nor does it disrupt the lipid bilayer of the proteoliposomes or interfere with the accessibility of magnesium. In conclusion, we demonstrated that compound $\mathbf{1}$ is able to inhibit the transport of two different vitamins and selectively inhibit the family of ECF proteins and not ABC transporters in general.

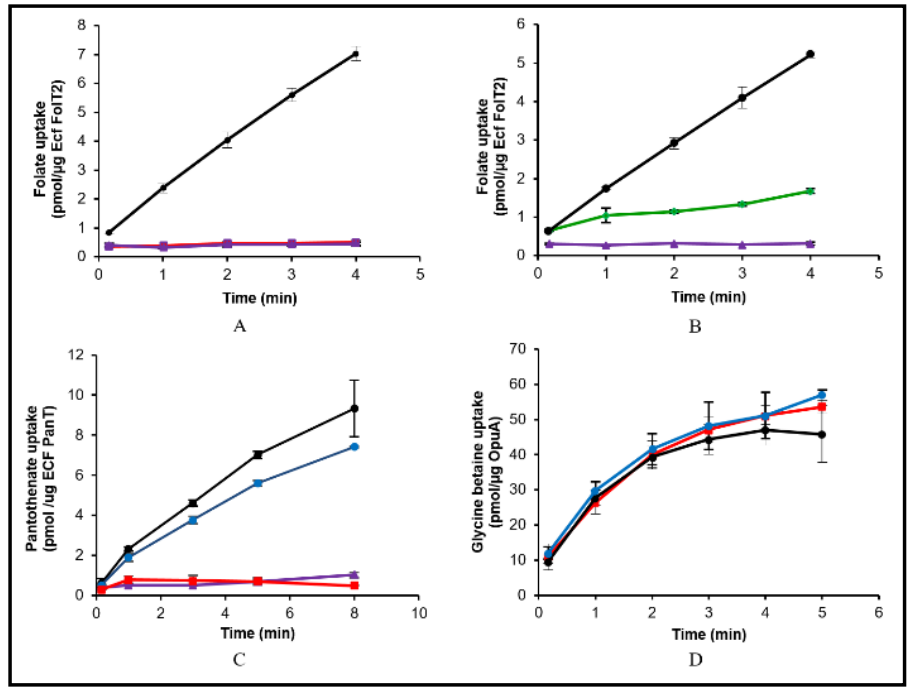


Fig. 3 Effects of compounds $\mathbf{1}$ and $\mathbf{2}$ on the transport activity of ECF transporters (ECF-FolT2 and PanT) and an unrelated ABC transporter (OpuA). (A) Folate uptake by ECF-FolT2 reconstituted in proteoliposomes filled with $5 \mathrm{mM}$ MgATP (black), 5 mM MgADP (purple) or $5 \mathrm{mM}$ MgATP plus $5 \mathrm{mM}$ of compound 1 (red), with a final DMSO concentration of $10 \%$ (v/v) in all experiments. (B) Same as in Panel A, including proteoliposomes filled with $5 \mathrm{mM} \mathrm{MgATP}$ plus $5 \mathrm{mM}$ of compound 2 (green). (C) Pantothenate uptake by ECF PanT in proteoliposomes filled with $5 \mathrm{mM}$ of MgATP (black), $5 \mathrm{mM}$ of MgADP (purple), $5 \mathrm{mM}$ MgATP plus $5 \mathrm{mM}$ of compound 1 (red), and a final DMSO concentrations of $5 \%(\mathrm{v} / \mathrm{v})$. The blue curve represents proteoliposomes filled with $5 \mathrm{mM}$ MgATP and no DMSO. (D) Glycine betaine uptake by OpuA in proteoliposomes filled as described in Panel C. The error bars represent the standard deviation of three independent measurements, except for the data in panel $\mathrm{C}$, where they represent the spread of the data from two independent measurements.

\section{Structure-activity relationship (SAR) study around 1}

Encouraged by the potency and drug-likeness of 1, we sought to confirm structural features that are critical for inhibition of the ECF transporter. The low resolution of the available crystal structure might lead to unreliable docking predictions approach; therefore we initiated a focused SAR study first aimed at exploring the role of 2hydroxybenzoic acid with a small set of compounds (13-21) prepared as described in Scheme S1. Removal of one group at the time yielded monosubstituted derivatives 13 and 14. Loss of inhibitory activity was observed in both cases, supporting the key role played by the salicylic acid portion. The reduction of the carboxylic acid to a primary alcohol, its methyl esterification and amidation yielded compounds 15-17. While compound $\mathbf{1 5}$ is twofold less potent than 1 ( $14 \%$ inhibition at a concentration of $250 \mu \mathrm{M})$, the replacement of the carboxylic acid with an amide (17) had no significant effect ( $27 \%$ inhibition at a concentration of $250 \mu \mathrm{M})$. Unfortunately, the solubility of $\mathbf{1 6}$ in the assay buffer is substantially decreased. Next, we turned our attention to the role of the hydroxyl group and found that its replacement with a methyl group (18) or methylation (19) reduced the inhibitory activity. By contrast, the finding that $\mathbf{2 0}$ is able to inhibit ECF activity with a two-fold higher potency compared to $1\left(20, I C_{50}=134 \mu \mathrm{M}\right)$ supported the notion that a sterically bulky carbamate moiety is well-tolerated.<smiles>[X]c1ccc(OCc2cccc3ccccc23)cc1[X]</smiles>

Table 1: Inhibitory potencies (IC 50 values and percentages of inhibition) of compound $\mathbf{1}$ and analogues 13-21 on ECF FolT-2.

\begin{tabular}{|c|c|c|c|c|}
\hline Cmpd & $\mathbf{X}$ & $\mathbf{Y}$ & $\begin{array}{c}\text { \%Inh.@250 } \boldsymbol{M M} \\
\mathbf{\pm} \text { S.E.M. }\end{array}$ & $\mathbf{I C}_{\mathbf{5 0}}(\boldsymbol{\mu} \mathbf{M})$ \\
\hline $\mathbf{1}$ & $\mathrm{OH}$ & $\mathrm{COOH}$ & $34 \pm 9$ & $282 \pm 108$ \\
\hline $\mathbf{1 3}$ & $\mathrm{H}$ & $\mathrm{COOH}$ & No inhibition & - \\
\hline $\mathbf{1 4}$ & $\mathrm{OH}$ & $\mathrm{H}$ & No inhibition & - \\
\hline $\mathbf{1 5}$ & $\mathrm{OH}$ & $\mathrm{CH}_{2} \mathrm{OH}$ & $14 \pm 17$ & - \\
\hline $\mathbf{1 6}$ & $\mathrm{OH}$ & $\mathrm{COOCH}_{3}$ & n.d. & - \\
\hline $\mathbf{1 7}$ & $\mathrm{OH}$ & $\mathrm{CONH}$ & $27 \pm 3$ & - \\
\hline $\mathbf{1 8}$ & $\mathrm{CH}_{3}$ & $\mathrm{COOH}$ & $17 \pm 3$ & - \\
\hline $\mathbf{1 9}$ & $\mathrm{OCH}_{3}$ & $\mathrm{COOH}$ & $27 \pm 10$ & - \\
\hline $\mathbf{2 0}$ & $\mathrm{NHBoc}^{*} \mathrm{COOH}$ & $76 \pm 4$ & $134 \pm 26$ \\
\hline $\mathbf{2 1}$ & $\mathrm{NH}_{2}$ & $\mathrm{COOH}$ & $8 \pm 5$ & - \\
\hline
\end{tabular}

as.E.M. derived from at least two experiments

${ }^{*}$ n.d. : not soluble under the conditions of the assay

After demonstrating that this chemical class interferes with the uptake of folic acid and pantothenate via inhibiting the ECF transporter in vitro (Fig. 3b-c), we investigated whether $\mathbf{2 0}$ might inhibit ECF activity in a cellular set up. Therefore, we developed a cell-based transport assay using E. coli MC 1061 heterologously producing ECF_FolT2 and evaluated the ability of $\mathbf{2 0}$ to interfere with the uptake of radiolabeled folic acid. As a control, we used $E$. coli MC 1061 that was not induced for expression. By analogy with the in vitro experiment, also in a cell-based assay, our compound $\mathbf{2 0}$ showed an inhibition of $93 \pm 1 \%$ at a concentration of $250 \mu \mathrm{M}$ (Table 2). Taken together these findings are in agreement with the proposed allosteric mode of inhibition and show that $\mathbf{2 0}$ indeed inhibits the ECF transporter. 
Table 2: Inhibition of radiolabeled folic acid in Escherichia coli MC1061 by 20 with and without induction of heterologously expressed ECF_FolT2.

\begin{tabular}{|l|l|}
\hline Indicator strains & \multicolumn{1}{c|}{20} \\
& $\%$ Inh. @250 $\mathrm{MM}$ \\
\hline Escherichia coli MC1061 ECF_FolT2 & $93 \pm 1$ \\
\hline Escherichia coli MC1061 & no uptake \\
\hline
\end{tabular}

The lack of uptake in the absence of heterologous expression (Figure $4 \mathrm{~A}$ ) indicates that there is no alternative transporter for folic acid that facilitates the uptake of it at nanomolar concentration. Lastly, we corroborated the allosteric mode of inhibition of $\mathbf{2 0}$ in the in vivo set up by using a 2.5 -fold excess of the vitamin substrate (Fig. $4 B)$, which did not affect the inhibition significantly.

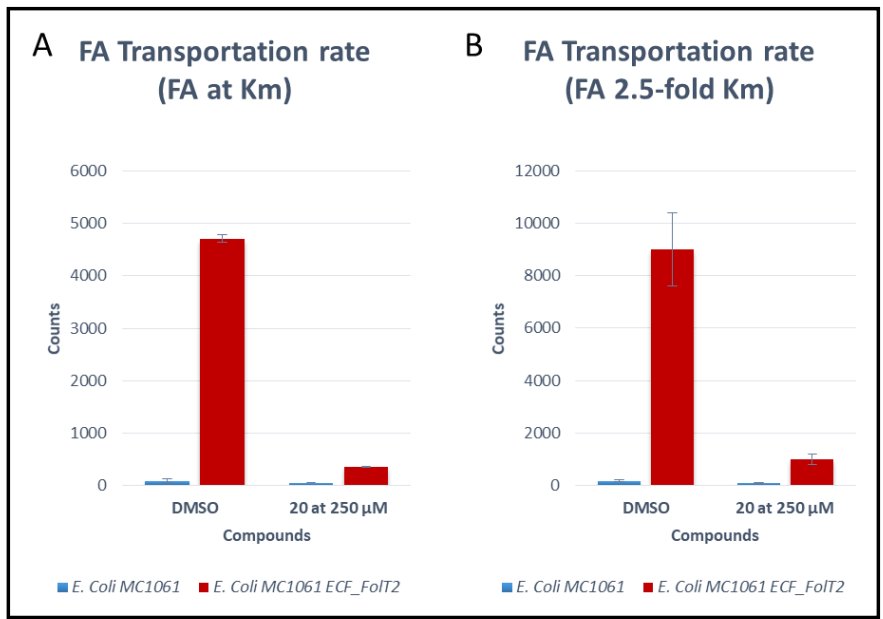

Fig. 4 A) Uptake of radiolabeled folic acid (concentration $50 \mathrm{nM}$ ) by Escherichia coli cells in presence and absence of compound 20 . The $E$. coli MC1061 strain does not show endogenous uptake of folic acid (blue bars), in contrast to the $E$. coli cells that heterologously express the ECF-FolT2 transporter from L. delbrueckii (MC1061 ECF_FolT2, red bars). At a concentration of at $250 \mu \mathrm{M}$, inhibitor 20 blocks the uptake of radiolabeled folic acid (concentration $50 \mathrm{nM}$ ) by $93 \%$. B) Uptake of radiolabeled folic acid (concentration $125 \mathrm{nM}$ ) in presence and absence of compound 20. Inhibitor 20 blocks the uptake by $88 \pm 2 \%$ at a concentration of $250 \mu \mathrm{M}$. The values represent the average of two independent experiments, and the error bars indicate the range.

These encouraging results prompted us to measure the effect of compound 20 on the human pathogen Streptococcus pneumoniae. This organism is auxotrophic for pantothenate and biotin and depends on ECF transporters for their uptake. Therefore, we predicted that inhibition of the ECF transporter would reduce its viability. Consistently, compound $\mathbf{2 0}$ inhibited growth of S. pneumoniae, but the MIC (minimum inhibitory concentration) was remarkably low compared to its in vitro potency (Table 3) presumably, because the uptake of more than one vitamin was affected. In fact, in the cell-based assay the effect of a diminished uptake of multiple micronutrients may have a synergistical impact on the cell survivability compared to the inhibition of uptake of only one micronutrient we monitor in the in vitro assay.

As a negative control, we used a Gram-negative strain that does not express the ECF transporter (E. coli K12) and expectedly, we did not observe an antibacterial effect of compound $\mathbf{2 0 .}$

Table 3: Antibacterial profile of compound 20.

\begin{tabular}{|l|l|}
\hline Indicator strains & $\mathbf{2 0} \mathrm{MIC}[\boldsymbol{\mu g} / \mathrm{mL}]$ \\
\hline Streptococcus pneumoniae DSM-11865[a] & 2 \\
\hline Escherichia coli K12 & $>100$ \\
\hline
\end{tabular}

[a]PRSP: Penicillin-resistant Streptococcus pneumoniae.

Compound $\mathbf{2 0}$ was tested for cytotoxic effects on a set of cell lines that includes HEK293 (human embryonic kidney cells), HepG2 (human liver cancer cells), and A549 (adenocarcinomic human alveolar basal epithelial cells). Notably, while 20 did not show any considerable cytotoxic effects in A549 cells at $100 \mu \mathrm{M}$ (Fig. 5), a moderate decrease in viability was observed in HepG2 and HEK293 cells. This effect was in the same range observed with 
the positive control doxorubicin at a 20 -fold lower concentration. The approximate IC 50 value of compound $\mathbf{2 0}$ in HepG2 and HEK293 cells was $\sim 100 \mu \mathrm{M}$ and thus much higher than the antibacterial effects observed in Streptococcus pneumoniae (MIC of $2 \mu \mathrm{g} / \mathrm{mL}$ ) leading to a good therapeutic window.

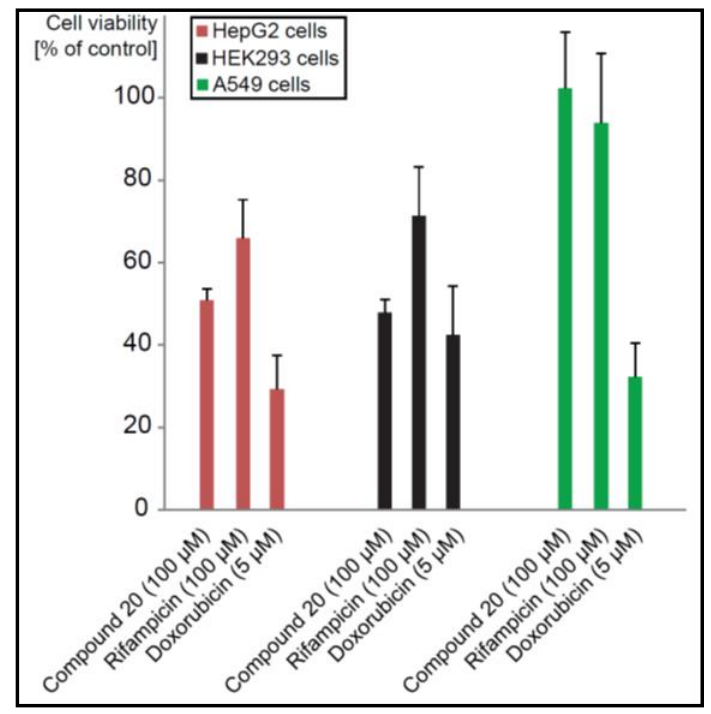

Fig. 5 Cytotoxicity determination of compound $\mathbf{2 0 .}$

Furthermore, we assessed the stability of compound $\mathbf{2 0}$ in human plasma and liver microsomes (Table S1) and found an adequate stability in plasma (20,t/2 $>2.5 \mathrm{~h}$ ) and metabolic stability $(\mathbf{2 0}$, hepatic Clint $<12 \mu \mathrm{L} / \mathrm{min} / \mathrm{mg})$. Thus, our results confirm compound $\mathbf{2 0}$ as the first molecular probe that may be used to investigate the role of the ECF transporters both in vitro and in cellulo.

Lastly, by using a fluctuation test we examined the ability of $S$. pneumoniae (strain D39V) to become resistant to compound 20. In contrast to the rifampicin control, where we could readily select resistant mutants (experiment was performed at 2-fold MIC), no mutants resistant to compound $\mathbf{2 0}$ (at 2-fold MIC) could be isolated, indicating a very low potential for resistance development against this type of ECF transporter inhibitors (Table S2).

\section{Searching for evidences of the action mechanism}

Although, all experimental data point to selective inhibition of the ECF transporters by the hit compounds, it was not possible to experimentally confirm their binding mode. Mutagenesis experiments in P2 pockets were inconclusive as they resulted in low uptake levels of the radiolabelled vitamin, indicating the importance of the selected amino acids in the mechanism of action (Fig. S30). In addition, extensive attempts to co-crystallize the inhibitors with the ECF protein, have been unsuccessful so far. Nonetheless, some of our previous experimental studies provide indications. Given that the compounds inhibit the uptake of both folic acid and pantothenate into the liposomes in the in vitro assay (Fig. $3 \mathrm{~A}-\mathrm{C}$ ) and, that two different S-components are involved in the transport of the two substrates, we suggest that the mode of inhibition does not involve high-affinity binding to the substrate binding pockets of the S-component. It should rather involve interaction with the ECF module of the transporter or with one of its interface with the S-component.

To further explore all possible binding pockets of the ECF transporter, we performed coarse-grained (CG) molecular dynamics simulations using the recent unbiased sampling approach ${ }^{31}$ based on the new version of the Martini force-field, called Martini $3 .^{32}$ The model has so far accurately predicted binding pockets and binding modes for pharmaceutical relevant targets such as nuclear receptors, GPCRs and kinases. Predictions of binding affinities are also possible, in case of sufficient sampling. ${ }^{31}$ The bacterial membrane model, ECF transporter and inhibitors were included in the system, which was simulated for a total of 0.3 milliseconds, allowing not only to explore all possible pockets, but also to capture competition with the membrane and enough association and dissociation events to estimate binding free energies $\left(\Delta G_{\text {bind }}\right)$. For transmembrane targets this is a crucial component that need to be considered but is neglected in docking approaches. The results indicated that the inhibitors not only can bind in pocket P2, but also in two additional pockets: at the entrance of P11, which is the main cavity of the S-component pocket, and in P9 which is a narrow, 
hydrophobic and partially hidden pocket at the interface between EcfT and the S-component (Fig. 6A and Figure $\mathrm{S} 30$ ). Although the binding to $\mathrm{P} 2$ is not so favourable, a negative $\Delta \mathrm{G}_{\text {bind }}$ in relation to water for compound $\mathbf{1}$ ( $\Delta \mathrm{G}_{\mathrm{P} 2-}$ water $=-11.6 \pm 1.1 \mathrm{~kJ} / \mathrm{mol}$ ) may explain this compound being found as a hit in the SBVS campaign. The entrance of $\mathrm{P} 11$ seems to be a more relevant pocket in relation to water than $\mathrm{P} 2$, with a $\Delta \mathrm{G}$ p11-water of $-13.1 \pm 0.4 \mathrm{~kJ} / \mathrm{mol}$. However, the competition with the membrane also makes this pocket less relevant, as the pocket is embedded in the lipid environment. Indeed compound 1 can easily escape as the $\Delta G_{p 11-m e m b}$ is $4.2 \pm 0.4 \mathrm{~kJ} / \mathrm{mol}$. In addition, this pocket varies between different S-components as each one of them binds a specific vitamin and is therefore not in agreement with the observed inhibition of multiple substrates.

Prediction of binding free energy in relation to water or membrane places P9 as the most probable binding

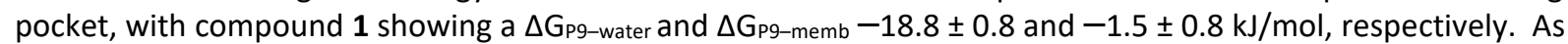
$\mathrm{P} 2$, the binding to $\mathrm{P} 9$ is also in line with the strategy of blocking the movements of the coupling helices taking place in the ECF module that are probably involved in the toppling and/or dissociation of the S-component. A representative pose of 1 in P9 (Fig. 6B) shows that certain polar/charged residues (like Ser 173 and Lys 102), that are not so near to this pocket in the crystal structure, can form a hydrogen bond network with the salicylic acid moiety, which suggests an important role for this substituent in the inhibitory action.

The comparison of $\Delta \mathrm{Gpg}$-water estimates of compound $\mathbf{1}$ with compounds $\mathbf{1 4}$, which shows no inhibition activity, and 20, the best inhibitor obtained in the study $\left(I_{50}=134 \pm 26 \mu \mathrm{M}\right)$ seems to reinforce the hypothesis that $\mathrm{P} 9$ is the most probably pocket. For pocket P9, the results nicely correlate with the inhibitory activity, while for the other pockets compound $\mathbf{1 4}$ emerges with highest binding affinity in relation to water. The proximity of P9 and its competition with the membrane may also explain the lack of inhibitory activity of $\mathbf{1 4}$ as its $\Delta$ Gp9-memb is unfavorable $(+8.2 \pm 1.41 \mathrm{~kJ} / \mathrm{mol})$, while compounds $\mathbf{1}$ and $\mathbf{2 0}$ show almost zero $\Delta \mathrm{G}_{\text {bind }}$ for $\mathrm{P} 9$ in relation to the membrane. As indicated by the water/partitioning, all compounds simulated tend to be located in the membrane. However, the removal of the carboxylate group (in relation to compound 1) makes compound 14 too hydrophobic. The replacement of the hydroxyl group in compound $\mathbf{1}$ for the NHBoc group in compound $\mathbf{2 0}$ seems to promotes additional hydrophobic interactions of the tert-butyl group with Leu 172 (Fig. 6C). At the same time, the hydrogen bond of $\mathrm{OH}$ group is mantained by the $\mathrm{NHBoc}$ group of compound $\mathbf{2 0}$. These combined effects possibly explain the higher affinity of 20.

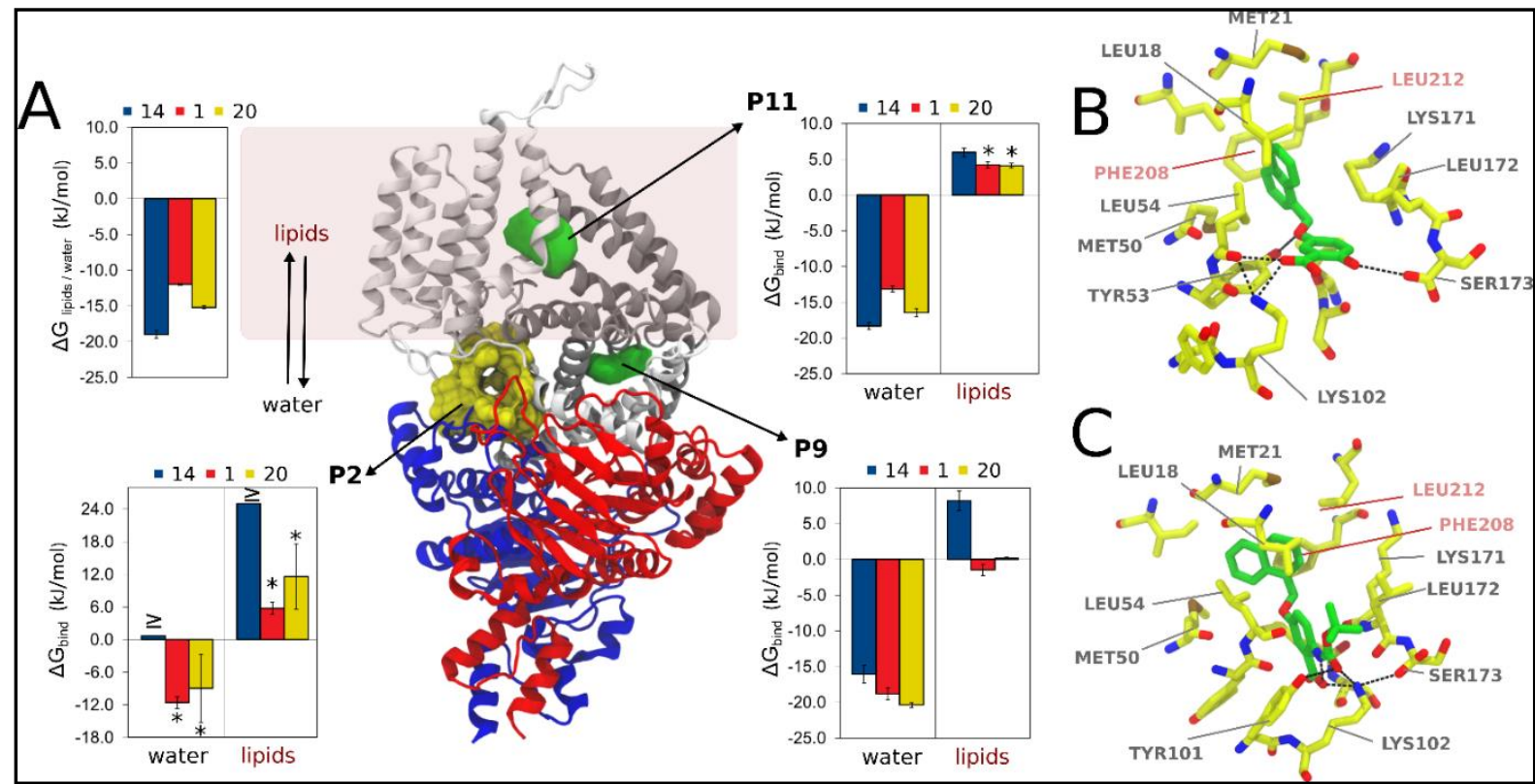

Fig. 6 Unbiased coarse-grained molecular dynamics simulations of inhibitors binding to ECF transporter. (A) Crystal structure of ECF FolT2 (PDB ID 5JSZ), in complex with EcfA and EcfA' shown in blue and red, EcfT in white and S-component in gray. Pocket P2, used in the structurebased virtual screening campaign is highlighted in yellow. Compound $\mathbf{1}$ densities obtained from the simulations are shown in green, which indicates the existence of two other pockets, P9 and entrance of P11 (pockets names according to Fig. S1). The green isosurfaces correspond to regions with high occupancy (100 times higher than compound $\mathbf{1}$ density in the membrane). As compound $\mathbf{1}$ binds only with low affinity to $P 2$, no high occupancy density is observed. The figure also shows bar plots with calculated binding free energies ( $\Delta G_{\text {bind }}$ ) of compounds 1 , 14 and $\mathbf{2 0}$ for pockets P2, P9 and P11 in relation to water and membrane. In the case of compound 14 in pocket P2, the binding events are so reduced that it is only possible to estimate the upper limit (indicated by " $\geq$ " in the plots). Estimates of the partitioning free energies of the inhibitors between water and lipids ( $\Delta \mathrm{G}_{\text {lipids/water }}$ ) are also show in the figure. (B) and (C) Representative poses of compounds $\mathbf{1}$ and $\mathbf{2 0}$ inside pocket $P 9$, respectively. Backmapped from the coarse-grained to the atomistic resolution. Some residues from EcfT (salmon) $S-$ component (gray) are highlighted, including the hydrogen bond network (black dashed lines). Hydrogen atoms have been omitted for clarity. 
This complete view including protein flexibility, and competition between environments and (hidden) protein pockets can only be properly estimated in the MD simulations as they consider all of these effects. ${ }^{33}$ Additional redocking calculations (starting from relaxed configurations from the simulations) performed now in P2, P9 and P11, did not provide new evidence for the preference for P9 in relation to the other pockets (Figure S32, Table S4). However, future experimental evidence is still necessary to confirm the binding pocket and provide the complete molecular mechanism involved in the inhibition of the ECF transporters promoted by our newly discovered compounds.

\section{Conclusions}

In conclusion, we report the identification of the hydroxy benzoic acid and phenyl furan chemical classes as the first inhibitors of ECF transporters. The focused SAR study around the salicylic acid scaffold provided a first insight into the shape requirements for fragment growing. We showed both in vitro and in cellulo that this chemical class has an allosteric mode of inhibition by inhibiting the ECF module and potentially binds to the ECF-T domain. Results from unbiased coarse-grained simulations were in agreement with the design strategy, showing that the ligand probably binds at the interface involving the S-component and ECF module. Although our experimental data strongly indicate that the ECF transporter is the target and that the design hypothesis is correct, future work must include more direct assessment of binding pocket and mechanism of inhibition of the inhibitors.

The 2-hydroxybenzoic acid is mandatory for activity whilst introduction of a carbamate function in the 2-position balances potency, metabolic stability and influences the growth of S. pneumoniae as shown by compound 20. The lack of cytotoxicity, the promising plasma and metabolic stability and the lack of resistance development observed under the conditions tested, establishes compound $\mathbf{2 0}$ as an excellent chemical probe to foster probe- and drug-discovery efforts in the field of the ECF transporters, which may open a new avenue and promising target in the antibacterial field.

\section{Acknowledgements}

A.K.H.H. and D.J.S. gratefully acknowledge funding from the Netherlands Organization for Scientific Research (ChemThem and VIDI grants). A.K.H.H. acknowledges funding from the European Research Council (ERC starting grant 757913) and the European Union's Horizon 2020 research and innovation programme (COFUND-ALERT, Grant No. 665250 ). D.J.S acknowledges support from the European Research Council (ERC PoC grant 812867). I.S received a scholarship from the Indonesia Endowment Fund for Education (LPDPLembaga Pengelolaan Dana Pendidikan, Departemen Keuangan, Republik Indonesia). Work in the Veening lab is supported by the Swiss National Science Foundation (SNSF) (project grant 31003A_172861), a JPIAMR grant (40AR40_185533) from SNSF, and ERC consolidator grant 771534-PneumoCaTChER. The authors thank Dr. Teresa Röhrig and Tabea-Catharina Schramm for the plasma and metabolic stability measurement, Jeannine Jung for the cytotoxicity evaluation, Dr. Daniel Krug and Patrick Haack for the analytical measurement, and H. Sikkema for glycine betaine uptake assays. The authors would like to thank also Viktoria Schmitt and Arnau Domenech for antibacterial testing. P.C.T.S. and S.J.M. acknowledge the National Computing Facilities Foundation (NCF) of The Netherlands Organization for Scientific Research (NWO) for providing computing time.

\section{References}

1 J. Ter Beek, A. Guskov and D. J. Slotboom, J. Gen. Physiol. 2014, 143, 419-435.

2 C. F. Higgins, Annu. Rev. Cell Biol. 1992, 8, 67-113.

3 G. Ames, C. Mimura and V. Shyamala, FEMS Microbiol. Rev. 1990, 75, 429-446.

4 A. L. Davidson, E. Dassa, C. Orelle and J. Chen, Microbiol. Mol. Biol. Rev. 2008, 72, 317-64.

5 A. Eudes, G. B. Erkens, D. J. Slotboom, D. A. Rodionov, V. Naponelli and A. D. Hanson J. Bacteriol. Res. 2008, 190, 7591-7594.

6 J. Song, C. Ji and J.Z.H. Zhang, Sci. Rep. 2013, 3, 3566.

7 J.A. Santos, S. Rempel, S.T. Mous, C. T. Pereira, J. Ter Beek, J.-W. Gier, A. Guskov and D. J. Slotboom, eLife $2018,7$.

8 F. Finkenwirth, F. Kirsch and T. Eitinger J. Bacteriol. 2013, 195, 4105-4111.

9 M. Majsnerowska, J. Ter Beek, W.K. Stanek, R.H. Duurkens and D.J. Slotboom Biochemistry 2015, 54, 4763-4766.

10 G. B. Erkens, R.P.A. Berntsson, F. Fulyani, M. Majsnerowska, A. Vujičić-Žagar, J. Ter Beek, B. Poolman and D. J. Slotboom Nat. Struct.Mol. Biol. 2011, 18, 755-760. 
11 M. Zhang, Z. Bao, Q. Zhao, H. Guo, K. Xu, C. Wang and P. Zhang, Proc. Natl. Acad. Sci. U. S. A. 2014, 111, 1856018565.

12 Y. Yu, M. Zhou, F. Kirsch, C. Xu, L. Zhang, Y. Wang, Z. Jiang, N. Wang, J. Li, T. Eitinger et al. Cell Res. 2014, 24, 267277.

13 Bao, Z.; Qi, X.; Hong, S.; Xu, K.; He, F.; Zhang, M.; Chen, J.; Chao, D.; Zhao, W.; Li, D.; et al. Cell Res. 2017, 27, 675687

14 D. A. Rodionov, P. Hebbeln, A. Eudes, J. Ter Beek, I. A. Rodionova, G.B. Erkens, D. J. Slotboom, M.S. Gelfa nd, A.L. Osterman, A. D. Hanson et al. J. Bacteriol. 2009, 191, 42-51.

15 S. Bousis, I. Setyawati, E. Diamanti, D.J. Slotboom and A. K.H. Hirsch, Adv. Therap. 2018, 233, 1800066.

16 N. K. Karpowich and D.-N. Wang, Proc. Natl. Acad. Sci. U. S. A. 2013, 110, 2534-2539.

17 D. J. Slotboom, Nat. Rev. Microbiol. 2014, 12, 79-87.

18 T. Wang, G. Fu, X. Pan, J. Wu, X. Gong, J. Wang, Y. Shi, Nature 2013, 497, 272-276.

19 I. Faustino, H. Abdizadeh, P.C.T.Souza, A. Jeucken, W. K. Stanek, A. Guskov, D. J. Slotboom, and S. J. Marrink, Nat. Commun., 2020, 11, 1-9.

20 L. J. Y. M. Swier, L. Monjas, A. Guskov, A. R. de Voogd, G. B.Erkens, D. J. Slotboom and A. K. H. Hirsch, Chembiochem $2015,16,819-826$.

21 L. Monjas, L. J. Y. M. Swier, A. R. de Voogd, R. C.Oudshoorn, A. K. H.Hirsch and D. J. Slotboom, MedChemComm 2016, 7, 966-971.

22 L. J. Y. M. Swier, L. Monjas, F. Reeßing, R. C. Oudshoorn, A. Aisyah, T. Primke, M. M. Bakker, E. van Olst, T. Ritschel, I. Faustino, MedChemComm 2017, 8, 1121-1130.

23 L. J. Y. M, Swier, A. Guskov and D. J. Slotboom Nat. Commun. 2016, 7, 11072.

24 A. Volkamer, D. Kuhn, F. Rippmann and M. Rarey, Bioinformatics 2012, 28, 2074-2075.

25 M. R. Berthold, N. Cebron, F. Dill, T. R. Gabriel, T. Kötter, T. Meinl, P. Ohl, C. Sieb, K. Thiel and B. Wiswedel, Stud. Classif. Data Anal. Knowl. Organ., 2007 th ed.; Springer, 2007.

26 BioSolvelT GmbH -. LeadIT. version 2.1.8; Sankt Augustin, Germany, 2014.

27 BioSolvelT GmbH -. SeeSAR (version 3.2), 2015

28 J. B. Baell, G. A. Holloway, J. Med.Chem. 2010, 53, 2719-2740.

29 R. F. Bruns and I. A. Watson, J. Med.Chem. 2012, 55, 9763-9772.

30 I. A. Watson, https://github.com/lanAWatson/Lilly-Medchem-Rules.

31 P.C.T. Souza, S. Thallmair, P. Conflitti, C. Ramírez-Palacios, R. Alessandri, S. Raniolo, V. Limongelli and S. J. Marrink, Nat. Commun. 2020, 11, 1-11.

32 P.C.T.Souza, R. Alessandri, J. Barnoud, S. Thallmair, I. Faustino, F. Grünewald, I. Patmanidis, H. Abdizadeh, B. M. H. Bruininks, T. A. Wassenaar, P.C. Kroon, J. Melcr, V. Nieto, V. Corradi, H.M. Khan, J. Domański, M. Javanainen, H. Martinez-Seara, N. Reuter, R.B. Best, I. Vattulainen, L. Monticelli, X. Periole, D.P. Tieleman, A.H. de Vries, S.J.Marrink Nat. Methods 2021, 18, 382-388.

33 P.C.T.Souza, V. Limongelli, S. Wu, Siewert J. Marrink, L. Monticelli Front. Mol. Biosci. 2021, 8, 657222, 1-9. 